\title{
Technique Development
}

National Cancer Institute

\section{Source}

National Cancer Institute. Technique Development. NCI Thesaurus. Code C15894.

Develop and evaluate new laboratory assays, behavioral methods, and statistical techniques for epidemiologic studies. (FMB) 